\title{
On the use of Kripke modality in language theory
}

\author{
K. HVIDTfELt NIELSEN \\ Institute of Language, Literature and Culture, University of Aarhus, Denmark
}

\begin{abstract}
Mit diesem Aufsatz soll demonstriert werden, dass das Phänomen Modalität neben seiner objektsprachlichen Existenz auch als deskriptives Mittel der Sprachtheorie verwendet werden kann. In Nielsen (2003) habe ich eine Sprachtheorie vorgelegt, nach der die semantischen Aspekte der natürlichen Sprache einen grundsätzlich unentscheidbaren Bereich bilden. Wie jede sprachskeptische Theorie muss auch die meinige mit dem bekannten Dilemma der Selbstreferenz zurechtkommen. Hier bietet sich die formale Technik der Kripke-Modalität als eine praktikable Lösung dar. Ich zeige, wie diejenigen sprachlichen Züge, die meiner Theorie zufolge für das Entstehen semantischer Unentscheidbarkeit verantwortlich sind, als vier formale Restriktionen eines modalen Kripke-Modells abzubilden sind (Irreflexibilität, Endlichkeit, inverse Wohlfundiertheit, strikte Linearität). Ich zeige weiter, wie sich diesem Modell eine formale Modalsprache zuordnen lässt, die das Modell und seine interne Struktur absolut entscheidbar denotiert. Da das Modell die relevanten Grundzüge meiner Theorie repräsentiert, mag die zugeordnete formale Sprache nun als ein Darstellungsmittel betrachtet werden, das die semantische Unentscheidbarkeit, von der es spricht, denotiert, ohne dabei dieser gleichzeitig zu verfallen.
\end{abstract}

\section{THE LANGUAGE SCEPTIC'S PARADOX - A PROBLEM EXPLAINED}

Many paradoxes arise from the linguistic combination of self-reference and negation. The Cretan claiming all Cretans to be liars provides one famous example. If the Cretan speaks the truth, he ought to be lying since that is the way of all Cretans; but then again, if he really lies as all Cretans are known to 
do, then he just may speak the truth, in which case he ought to be lying etc. Paradoxical experiences with language often lead to scepticism about the cognitive values of language. A century after the Cretan Epimenides disowned his fellow islanders, the sophist Gorgias launched a general attack on knowledge. Nothing exists, and should it exist, we could not know it, and should we be able to know it, we would be unable to say what we knew. Man is not adequately equipped to have knowledge. Language is no adequate instrument for expressing knowledge.

With Gorgias, epistemic and linguistic scepticism fused. There have been many attempts to silence the sophist suspicion, but none that has proved definite, or even broadly convincing. However, there is a problem with theoretically expressing and defending the position of epistemic language scepticism. Whoever tries, runs into a paradox, not unlike that of Cretan liars. Perhaps, the theoretical aspect of what happens when the attempt is made, is best explained in terms of consistency. Suppose that a language sceptic wants to argue the claim that all texts of natural language are inherently inconsistent. We need two things for the paradox to materialise: negation and self-reference. In our case, negation is inherent in the very property of inconsistency, and self-reference is achieved by applying the claim of inconsistency to the very text of natural language arguing the inconsistency of all natural language texts. Now, if the sceptic's arguments are consistent, he produces a text of natural language that is not consistent with his position as a sceptic, and so his arguments have disproved his language scepticism. On the other hand, should the sceptic's text prove to be inherently inconsistent, the sceptic will have produced a text that, albeit consistent with his scepticism, through its internal inconsistency will fail to prove its point. Under inconsistency nothing can be proved.

Cretans become liars by telling the truth and truth-tellers by lying. Language sceptics become inconsistent by consistently arguing their position, and consistent by inconsistent arguments. Cretans and language sceptics have an equal share in paradoxical speech. Or so it seems; for on closer consideration, one may detect a difference between the two kinds of paradoxes. Cretans can neither tell the truth, nor lie about their lying. If they could, there would be no paradox. It is a different story with language sceptics. 
Like Cretans, language sceptics know of two situations: they may consistently or inconsistently argue the inconsistency of all language texts. But it is only the former situation that is truly paradoxical. Just as Cretans fail to succeed in speaking truthfully about their lying, no language sceptics consistently manage to argue the inconsistency of all language texts. For if they could, they would have done what they had proved to be impossible (argued consistently). Hence the smack of paradox. But the second situation is perfectly in order: Naturally, language sceptics may inconsistently argue the inconsistency of natural language - that is, if you are ready to accept the notion of inconsistent argument. If Cretans lie about their lying, they tell the truth, and so the paradox starts rolling. But language sceptics arguing the inconsistency of all natural language argument, do not thereby get their linguistic behaviour entangled in paradoxical speech. As most language users know, inconsistent argument is an all too natural species of human communication. According to sceptics, the only one that man has access to at all.

When Cretans speak, they create a paradoxical situation between two symmetric positions, truth and falsity. With language sceptics, two asymmetric kinds of consistency are involved, one pertaining to the relationship between the sceptic and the text, another pertaining solely to the text. Let us call the former kind of consistency performative, and the latter textual. Formally, the language sceptic should know of two situations, one combining performative inconsistency with textual consistency, and one combining performative consistency with textual inconsistency. In real life, however, only the second combination is possible. Through the very notion of textual consistency, language sceptics are not likely to find themselves in a situation where they have become performatively inconsistent by consistently arguing their case. No one can consistently argue the inconsistency of all natural language texts. So languages sceptics, we may say, are better off than Cretans. Since it is performatively consistent for language sceptics to be textually inconsistent, it is not the blemish of paradox that language sceptics need to fear.

In 2003, I presented a theory of language scepticism² ${ }^{2}$ In the theory, two strands of reasoning are combined. The first may be characterised as the attempt to read Spinoza's Ethica as a forerunner of analytic language theory. The second is indicated by the subtitle of the book: Toward a Formal Theory of Consistent 
Language Scepticism. According to my interpretation, Ethica is organised around a paradox of language scepticism quite similar to that explained above. I argue that Spinoza discovered that his theory of affects applied to natural language. The use of natural language is basically an affective affair, and since affects, under Spinoza's interpretation, distort cognition, no text of natural language can produce cognition. Assuming that Spinoza did nourish cognitive aspirations on the part of his Ethica ${ }^{3}$ I further argue that Spinoza's reasons for choosing a geometrical format for his text originated in the belief that he could thereby immunise his own text from the cognitive defects otherwise befalling all kinds of natural language. The idea was bold, but at Spinoza's time, the formal resources of logic were not sufficiently developed to make the plan work.

Spinoza tried to escape the paradox of language scepticism, not by accepting inconsistency for his reasoning, but by disciplining his arguing through logic. As documented $^{4}$ many times, Spinoza did not accomplish his aim. In my opinion, Spinoza was hampered not only by the insufficient state of logic at his time; the whole idea of making natural language consistent by artificial means is misdirected. No use of natural language, however disciplined by geometry or logic, can, in my opinion, consistently demonstrate the inconsistency (with Spinoza: the affectability) of all texts of natural language. Consistency, as I suppose, is not to be had in natural language. A theory effectively aspiring to be consistently arguing the inconsistency of all texts of natural language is therefore well advised to forgo the use of natural language when ultimately setting out its points.

For the rest of this paper, I shall have nothing more to say about Spinoza's heroic fight with language. Instead I shall focus on explaining what I did in order to escape the theoretical dilemma of language scepticism. Sceptics leave paradoxes behind when they speak inconsistently about the inconsistency of natural language. But obviously, inconsistent speech is not very convincing that is, if we notice. When needed or convenient, we often accept understandability for consistency. So, the real challenge for the ambitious sceptic is to find a way of arguing or presenting a theory of language scepticism satisfying effective criteria of consistency. The second part of my book was dedicated to that task. 
In my brand of language scepticism, natural language equals usage. Quite generally, I describe usage as a change-inducing mechanism, operating in time according to deterministic laws. Usage effects changes in those engaged in usage. These changes are both mental and cerebral. What happens on the mental side, I call meanings. Meanings change by being activated (used). These changes are undecidable in the sense that no mechanism can be devised that would enable us to identify the meanings changing. It is another story with the brain, but that is beyond the point here. My theory is solely about meanings.

Users are either producers or recipients. There is no way to effectively decide what kinds of change that producers think their usage might effect in others (or in themselves), just as it is beyond our knowledge to determine what recipients believe the usage they receive (and process) to have effected in themselves. The intentions of users may be guessed at, but never, effectively, known. There is only one way of making usage decidable, and that is by tying all parts of usage, word by word, structure by structure, effectively to observable objects. The scientific usage of natural language may under certain well-controlled circumstances come within tolerable reach of this ideal. But normally, natural language usage functions according to quite different parameters.

I divide my theory in two parts: in the first half, I lay out, in normal scholarly English, the arguments and the evidence that sustain my theory. I write in the professional language of analytic theory, but my intentions are different from those, I gather, normally nourishing academic speech. I write with a view, not to proving any points (that would be openly inconsistent with my scepticism), but to changing readers' attitude to language (usage) in the sceptical direction I promote. Naturally, readers are, and will be, free to read my text with very different intentions. There is little in my interpretative or argumentative style to surprise or alienate professional readers. They may, for instance, choose to disregard my repeated indications of sceptical intentions, should they so want. Readers may even credit me with having proved the sceptic's case beyond any reasonable doubt. Being a sceptic, I would obviously have to disagree on the latter point.

For the second half of my book, I hope for readers made, by the first half, sufficiently susceptible to language scepticism in order to accept the need for 
decidably consistent theory. Yet less will also do. The second half is meant to interest not only persuaded sceptics, but also linguists or language theorists merely curious about the issue of consistency.

After having motivated readers to embrace or consider the position of language scepticism, I start out by devising a theory capable of expressing, in a totally consistent and decidable manner, my crucial points about the undecidabilities of meaning change. Like many scientists grown weary with the unruliness of natural language (if for slightly other reasons (see below)), I turn to formal languages. More precisely, I direct my search for expressive clarity to the branch of formal logic known as modal logic. Here I focus on the kind of model theory named after the logician Kripke. My idea is to find a modal Kripke model that I can regard as a formal representation of my views of natural language meaning change. How this is done and the consequences will be the subject of the remaining part of this paper. Perhaps, I should emphasise that my presentation of modal model theory is solely meant for linguists and language theorists. Logicians interested in seeing how their techniques may be applied outside the field for which they were designed, may find the presentation illuminating, but they will meet with little that will appeal to their professional creed or skills.

\section{DECIDABILITY - A CONCEPT EXPLAINED}

Historically, and technically, decidability is a formal concept. It emerged out of Gödel's spectacular findings in $1931^{5}$. Much to the surprise of mathematicians and logicians, Gödel discovered that not all formal systems are decidable. I shall not bother with the details here. I have discussed them in length in Nielsen (2003). However, once we know that some specific formal system, a formal language, is decidable, then we know that we can fully control our use of that language. In a moment, I shall extend the notion of decidability to non-formal areas as well, but the basic idea, both in formal and non-formal decidability, is that of possessing a purely mechanical procedure for determining, in an absolutely rigorous way, on some precisely formulated issue (often one that can be answered with a yes or a no).

Let us distinguish between two faces of formal decidability: a grammatical one and a referential (semantic) one. Logicians normally speak of decidability only in connection with grammar. What I have chosen to call referential (semantic) 
decidability is certainly a formal concept (in fact two), albeit rarely referred to under that heading ${ }^{6}$. My deviation from standard practice is more than terminological; it has to do with a restriction that I impose on my use (and understanding) of formal systems. In Nielsen (2003), I worked only with formal systems that can be realised in some physical medium, be it graphematic, electronic or whatever. Logicians would oppose such narrowness. Most of the things they want to do in and with formal systems can only be done when formal systems are unfettered by physical restrictions. Of course, I do not want to dispute the legitimacy or the necessity of the logicians' choice. There would be little left of their profession were they debarred from working with abstract systems. However, my interest in formal logic is very different from that of the professional logician. To me, a language theorist, formal logic is more - or perhaps I should say less - than a mathematically sophisticated branch of philosophy. I consider formal logic a reservoir of techniques to be freely used in the service of other disciplines. Not all of formal logic is decidable. However, when reduced to physical (and finite) dimensions, formal techniques shrink into decidable (and logically trivial) standards.

In the world of physical systems, decidability is no longer a purely formal concept, but takes on a more universal feature. In Nielsen (2003), I have argued that a Turing machine, reduced to finite dimensions, encapsulates the essence of what, in a physical system, one would like to understand by decidability. A procedure, a rule, a set is decidable if there is a purely mechanical way of determining the outcome of the procedure, the effect of the rule, or the extension of the set.

With these qualifications, one may give the following explanation of grammatical and referential decidability as applied to physically restricted formal systems ${ }^{7}$. A formal system consists of a (finite) vocabulary, meaning and denoting nothing, together with a grammar. A vocabulary may typically contain single words or letters (of various categories), operators (of various categories) and auxiliary signs. By means of the grammar, two sets of constructions may be defined: (1) the set of well-formed expressions, (2) the set of proofs. A proof is a special sequence of well-formed expressions. The last well-formed expression of the proof is called a thesis. One should be careful not to associate any special prestige to the grammatical category of theses. From the view-point of grammar, 
theses are just expressions complying with certain rules of construction. A formal system, then, is said to be grammatically decidable if any combination of elements from its vocabulary can be uniquely (and mechanically) parsed ${ }^{8}$. All the formal systems discussed in this paper will be grammatically decidable.

When logicians speak of proofs they are either referring to grammatical proofs of the kind just indicated ${ }^{9}$ or to special insights or procedures transcending the capacities of grammars. Most logicians agree on which procedures to accept and which to disown ${ }^{10}$. The matter is too complicated to be dealt with here. In this context, where logic has been reduced to a physical format, let us content ourselves with demanding that all procedures involved in non-grammatical proofs be as decidable as their grammatical cousins ${ }^{11}$.

What I have chosen to call referential (semantic) decidability is mostly dealt with by logicians under the headings of soundness and (semantic) completeness ${ }^{12}$ respectively. It is rather limited what can be done with a formal system alone apart from studying its grammatical features. If you want to extend the use of formal systems for instance by using it as a language with which to speak of other things (eventually itself), you must supplement the formal system with the kind of formal structure that logicians call a model. Logicians, of course, think of models as abstract, mostly set-theoretical, constructions. Abiding by my materialism, I demand of models that they should always, in principle, be susceptible to physical realisation or computer simulation. But whatever the stuff that models are made of, basically a model is a collection of those items that one uses a formal system to refer to (speak of).

Logicians study the relationship between formal systems and models under the two aspects alluded to above. Both involve the notion of formal truth. From our view-point, we may think of formal truth in terms of reference or denotation ${ }^{13}$. If an expression of a formal system is formally true in a model, then that expression will have what I shall call a positive reference in that model. To establish a positive reference, one, normally, proceeds by first determining the reference of the single words in one's vocabulary, and then going on to decide what the referential effects of the grammatical rules are. An expression has positive reference, then, if the grammatical rules allow for the words of the expression to refer to items in the model. 
Let us take a simple example. Suppose we had, in the vocabulary of some formal system, the word $\alpha$, and $\alpha$ referred to item A in some model. And suppose we had, in the same vocabulary, the operator $\neg$, along with a grammatical rule for using $\neg$ to the effect that $\neg \alpha$ was a well-formed expression. And finally, suppose that the referential effect of this grammatical rule should be to reverse the referential effect of the expression immediately after the operator. In this case $\neg$ would reverse the positive reference (the formal truth) of $\alpha$ into a negative one in the model. Notice that positive and negative references (formal truth and falsity) only make sense when viewed in relation to some specific model (or some class of models).

As might be clear from my explanation, all formal words will normally have a positive reference in some suitable model. This is seldom an agreeable situation to work with. So, in order to avoid this effect, logicians often prefer to work with systems that have no such words. Logicians call words with a fixed positive reference constants. The opposite of constants they call variables. One may think of variables as formal words that have not yet received some fixed positive reference. They are sort of temporary place-holders for future reference.

One can achieve the same effect by generalising over reference. Suppose we had a formal system with only two formal words, $\alpha$ and $\beta$, and likewise a model with only two items A and B. We might then wish to establish the maximal number of different ways in which the words of the system could be referentially combined with that model. When logicians (and mathematicians) generalise, they often include the empty possibility in the totality of items that they generalise over. Since, in this case, there is a perfectly good reason for adopting the logician's view-point, let me introduce the notion of an empty reference. Let us say that a formal word (of some system) has an empty reference if there is no item in the model to which the system refers. Let us call empty reference cenonymy ${ }^{14}$. By empty reference I am referring to a special case of negative reference. For convenience, I shall adopt the mathematician's practice and denote the empty element by $\varnothing$.

If we now, armed with cenonymy, ask how many different ways in which we may establish reference for $\alpha$ and $\beta$ in some model containing only A and B (plus the spooky $\varnothing$ ), we get two different answers according with our generosity 
with synonymy ${ }^{15}$. The two situations may be illustrated simply by listing the possible pairs:

1. Without synonymy: six possibilities: $[(\alpha, A),(\beta, B)],[(\alpha, A),(\beta, \varnothing)],[(\alpha, B)$, $(\beta, A)],[(\alpha, B),(\beta, \varnothing)],[(\alpha, \varnothing),(\beta, A)],[(\alpha, \varnothing),(\beta, B)]$

2. With synonymy: nine possibilities: $[(\alpha, A),(\beta, B)],[(\alpha, A),(\beta, \varnothing)],[(\alpha, A)$, $(\beta, A)],[(\alpha, B),(\beta, A)],[(\alpha, B),(\beta, \varnothing)],[(\alpha, B),(\beta, B)],[(\alpha, \varnothing),(\beta, A)],[(\alpha, \varnothing),(\beta, B)]$, $[(\alpha, \varnothing),(\beta, \varnothing)]$

Either list represents a special way of generalising over reference. The common idea is that of running through all admissible combinations between words and referents. In the former case, we found six different ways of attaching reference to our formal system, in the second nine. When we think of the relationship between a formal system and a model in this generalised way we say that an expression has valid reference (is valid) if it has a positive reference (if it is formally true) in any of the possibilities for combining words with referents in a model. When the relationship between a formal system and a model is conceived in this generalised fashion, the model is often called a frame $e^{16}$.

With the concept of a frame we are finally ready to understand the basic idea behind referential decidability. As previously stated, logicians address this issue under two headings: soundness and completeness. A system is said to be sound with respect to a frame if all theses of the system have valid reference on that frame $^{17}$. With completeness, the condition is the other way around: a system is said to be complete with respect to a frame if all expressions with valid reference on that frame are theses of the system. Having generalised over reference, we may be sure that no single words will have valid reference on a frame. The concept of cenonymy took care of that, as you may care to check in the two versions of generalised reference listed above.

So, what the idea behind referential decidability amounts to is that it enables the user of a formal system to establish an identity relation between the theses of the system and the expressions having valid reference on some frame. If a formal system is referentially decidable with respect to some frame, then we may be sure that all its theses, and all its theses only, will refer to items on that frame. Logical languages are regularly studied for the features of decidability. Once a new kind of logical language has been designed (or discovered) logicians check 
whether this new language complies with the strict demands of decidability. Most do, some do not. On my restricted view of logic, one can always tell whether or not some formal system is referentially decidable with respect to some frame. In real logical life, things are often much more complicated.

\section{DENOTING MEANING - A METHOD EXPLAINED}

I advocate a theory of meaning holding natural language meanings to be inherently undecidable. We can neither identify nor define natural language meanings. They change by being used, and since they exist only during and in usage, there can be no place from which meanings could be observed and identified which would not itself effect meaning change ${ }^{18}$. Were I now to hold that the meaning changes to which my theory (the reading of it) might give rise would have the identity and definitional force needed to support a theory, I would, as previously stated, be performatively inconsistent. I would demand something from my own usage that I deny to all other usage.

But meanings, one may counter, are hardly the stuff that proper theories should be made of. A theory, worth its salt, is basically a text, an ordered sequence of sentences with controllable references, and should be measured by its contribution to our grasp of the items referred to by the theory. However, the shift of focus from meanings to texts and referents does not make my theory fare any better as far as its main issue is concerned. Clearly, there are many aspects about usage that may be denoted in a sufficiently controllable way in order for professionals to assess the correctness of some theory on these points, but it should be equally clear that the meaning aspect will not be one of those. When there is nothing to identify, there is nothing to denote or to control.

Above, I discussed the general case of language scepticism. In the special case of meaning scepticism, such as presented here, the notion of textual inconsistency may now be given a more precise turn. The sceptic writing a theory about a subject that according to that theory defies identification confronts the challenge of writing about something which by hypothesis eludes reference. Reapplying a term previously introduced, we may say that meaning sceptics, in addition to the general plight of paradox, face the dilemma of theoretical cenonymy. They have to write about something that cannot be referred to at all ${ }^{19}$. 
In Nielsen (2003), I made the distinction between meanings (the plural) and meaning sui generis (the singular). By the plural I wanted to refer to the individual members in the class of those phenomena that one cannot, according to my theory, refer to individually. When using the genetic singular, I had a different referent in mind. I was thinking less of specific meanings occurring than of something which could also have been called the general structure of meaning. I argue that meanings are systematically undecidable, but I likewise contend that their undecidability happens under circumstances or in a mould that can be precisely and decidably described. What meanings are, is undecidable, but what makes them so need not therefore defy description.

Shifting the focus of my theory from meanings (the plural) to meaning (the singular) helped me reduce the problem of cenonymy to that of finding decidable means of denoting meaning. I strongly doubt that texts of natural languages can be so disciplined as to be grammatically and referentially decidable $^{20}$. In my opinion, no one really knows. Of course, my suspicion would be immediately disproved the moment someone produced just one single instance of a grammatically and referentially decidable text of natural language. But as long as no such specimen has ever come to my attention, I abide by my scepticism. If undecidability could come in degrees then I would assume for natural languages an ascending scale with grammar at the bottom and meaning at the top, and with reference somewhere in between.

Like me, also natural scientists distrust natural language. They have long ago given up pinning their professional faith on the referential decidability (or only reliance) of the natural languages. Instead they turn to mathematics or other formal languages when precision is in demand. As should be clear by now, I fully subscribe not only to the tacit disparagement of natural language inherent in the practices of natural science, but also to their choice of alternatives. So instead of trying to make natural language fit a controllable mould (Spinoza's way), I have chosen to use only completely formal and artificial techniques in my quest for referential decidability.

From my natural language presentation of the structure of meaning (the singular) I extracted the following four main characteristics of meanings (the plural). 
1. Natural language meanings are unrepeatable - no two occurrences of natural language meaning can be identical.

2. The number of meanings occurring, in some specific group of users, are finite.

3. Natural language meanings are determined by causal factors - meanings occur beyond our conscious control (or what we experience as conscious control is among the effects of meanings occurring).

4. Natural language meanings are temporal by nature - meanings occur in time.

Having thus reduced the general features of meaning to a tolerably tractable standard, I made the decisive move to formal techniques. For various reasons, I had become interested in the formal structures known as Kripke frames. Remember my approach to formal techniques. I use them as if they were physical procedures. So, I simply set out to investigate whether there among the rich variety of different Kripke frames could be a physically constructible one that could instantiate all four characteristics of meanings just indicated. As it turned out, my search was successful. I ended up with quite an interesting specimen. In the next and final section, I shall report some of the details of my findings.

\section{DECIDABLY DENOTING - A TECHNIQUE EXPLAINED}

In formal systems, modality is introduced by adding to the vocabulary of a nonmodal system the two operators box and diamond, $\square$ and $\diamond$. Actually, one of these will do, but for the sake of perspicuity I shall use both. Form the viewpoint of formal systems neither box nor diamond means anything; they are just inscriptions obeying grammatical rules. One often refers to the two operators as necessity and possibility, respectively, but one should be careful not to attach any particular importance to these names as far as formal systems are concerned. It is another matter when formal systems are used for referential purposes. Then the question arises of how to interpret well-formed expressions containing either operator.

In 1963, the logician and language theorist Kripke published a paper showing how to interpret so-called propositional modal systems in a strikingly simple and elegant way (Kripke 1963[1971]). The method has later been expanded to predicate modal systems as well, but, perhaps inevitably, not without losing 
some of its simplicity in the process. Predicate modal logic is a notoriously complicated affair, making it all the more reasonable to be pleased with the fact that we shall only have to deal with propositional modal systems (and their formal referents).

In section 1, I gave an informal explanation of how non-modal formal systems may be used for referential purposes. I showed how to define the notion of referential decidability (soundness and completeness) in terms of generalised models, also called frames. Basically, the same definition applies to modal formal systems as well. However, since in this section the emphasis will be on explaining the actual workings of formal reference, I shall set out the mechanism combining systems with models and frames once again, but now in a more detailed fashion.

Logicians often speak of reference in terms of interpretation. An interpretation is simply a model; thus, a frame is a generalised interpretation. Since models for ${ }^{21}$ modal systems are more complicated than those for non-modal systems, I start by explaining the simpler case. Non-modal systems are interpreted by defining a function from the expressions and the grammar of a formal system to items of the model. Formally, a model for a non-modal system is defined as the pair $<\mathrm{D}, \mathrm{V}>$. D denotes the domain of discourse that the expressions of the system refer to. $\mathrm{V}$ is the name of the function mapping expressions on to elements of $\mathrm{D}$. The interpretation of non-modal operators is defined in terms of certain truthconditional manoeuvres. In section 1, I gave an example of how the negation operator may be interpreted.

Modal systems call for a more elaborate interpretation (model). We start by dividing the domain of discourse into a multitude of different domains, each called a world. By using this terminology, Kripke obviously wanted to hark back to Leibniz's famous metaphysics of possible worlds. The idea is that we think of a possible world as a full description of some alternative to what we believe the actual world to be. Naturally, Kripke's definition of a possible world is completely formal. You need not believe in possible worlds in order to work with Kripke semantics. Some people, like me, prefer to think of a world solely as a collection of physical items. Whatever the case may be, in a Kripke model, the items to be spoken of by means of the formal system do not make up a uniform collection, but are grouped together in different sets or worlds. We therefore 
need a structure combining these worlds. Kripke showed that this job could be done simply by means of the dyadic relation R. By imposing varying conditions on $\mathrm{R}$ different structures would arise. It sounds like such a simple construction, but as the literature has shown, the dyadic R often gives rise to an amazingly rich variety of very sophisticated structures.

One of the conditions that may be placed on $\mathrm{R}$ is that of symmetry, but unless otherwise stated $\mathrm{R}$ is asymmetric. Another way of saying the same is to call $\mathrm{R}$ oriented or, as customary, to speak of $\mathrm{R}$ as an accessibility or successor relation. If two worlds, $\mathrm{w}$ and $\mathrm{w}^{\prime}$, are connected by $\mathrm{R}$, in that order, then the relation runs from $\mathrm{w}$ to $\mathrm{w}^{\prime}$. One normally denotes this relation as $w R w^{\prime}$, and $w R w^{\prime}$ is often circumscribed by saying that $\mathrm{w}$ has access to $\mathrm{w}^{\prime}$, or that $\mathrm{w}^{\prime}$ is the successor of $\mathrm{w}$. Formally, a Kripke model is the triple $\langle\mathrm{W}, \mathrm{R}, \mathrm{V}\rangle$ with $\mathrm{W}$ being a set of worlds, $\mathrm{R}$ a dyadic relation, and $\mathrm{V}$, as before, a function mapping expressions of a formal system on to the elements in the worlds of $\mathrm{W}$.

A modal expression is always to be interpreted relative to some world $\mathrm{w}$. The two modal operators are interpreted in terms of R. If $\alpha$ is some expression (or formal word) of some formal system, then $\square \alpha$ is interpreted by the stipulation that $\square \alpha$ is true (has a positive reference) relative to $\mathrm{w}$ if $\alpha$ is true (has a positive reference) relative to all worlds $\mathrm{w}^{\prime}$ accessible from $\mathrm{w}$. And $\diamond \alpha$ is interpreted by the stipulation that $\nabla \alpha$ is true (has a positive reference) relative to $\mathrm{w}$ if $\alpha$ is true (has a positive reference) relative to at least one world $\mathrm{w}^{\prime}$ accessible from $\mathrm{w}$. As may be seen, modal operators introduce an aspect of quantificational logic also when the formal system in question is purely propositional ${ }^{22}$.

In principle, there is only one system of non-modal propositional logic. There are many ways of setting up its axioms (that is, its basic theses), but they all produce the same system. With modal systems, things are more complicated. As new modal axioms are added to a system, different modal systems appear ${ }^{23}$, and since there seems to be no upper limit to the number of new axioms that can be added, modal systems make up an ever expanding class of formal systems.

It was for this multitude that Kripke designed his new way of interpreting. Kripke showed that one could obtain referential decidability for a great number of grammatically decidable modal systems simply by imposing special 
conditions on the dyadic relation $\mathrm{R}$ of the models for particular systems. If a modal system is grammatically decidable, then there exists a totally mechanical way of identifying those expressions which are theses. By referential decidability one denotes a situation where the theses of some system are exactly the same as those expressions that have valid reference (are valid) on some frame. So by means of grammatical and referential decidability, theses and valid expressions will be uniquely identified. We know that a frame is just a generalisation over some class of models. With the notation introduced in this section, this idea can now be given a more formal turn: suppose we had some Kripke model $\mathrm{M}=$ $<\mathrm{W}, \mathrm{R}, \mathrm{V}>$. We may now think of all the models differing from $\mathrm{M}$ only in the way in which $\mathrm{V}$ is defined between expressions and items of the worlds of $\mathrm{W}$. All these models (together with the initial $\mathrm{M}$ ) would make up exactly that class over which we generalise in order to get a frame. One indicates the result of this process simply by letting out the mapping V. Thus, a Kripke frame is the pair $<\mathrm{W}, \mathrm{R}>$, where $\mathrm{W}$ is a set of worlds, and $\mathrm{R}$ some dyadic relation.

Let me give a few examples of how referential decidability between modal systems and a frame is achieved by imposing suitable restrictions on $\mathrm{R}^{24}$. If you add the axiom known as $\mathrm{D}^{25}$ (for deontic): $\square \alpha \rightarrow \diamond \alpha$, to the non-modal propositional system (remember: there is only one), you get a modal system (also known as D) which may be proved to be grammatically and referentially decidable with respect to a frame where $\mathrm{R}$ is defined as follows: for all $\mathrm{w}$ in $\mathrm{W}$, there exists at least one $\mathrm{w}^{\prime}$ in $\mathrm{W}$ such that $\mathrm{wRw}^{\prime}$. This condition is often called seriality, and it has the effect of imposing on $\mathrm{W}$ a structure that is characterised by having no ends. There is no $\mathrm{w}$ that does not have access to at least one more $\mathrm{w}^{\prime}$. Seriality may be realised either by allowing $\mathrm{W}$ to be infinite or by imposing a circular structure on W. Naturally, materialists like me prefer the latter solution.

Another simple example would be the axiom known as $\mathrm{T}^{26}: \square \mathrm{A} \rightarrow \mathrm{A}$. When added to the propositional system, a modal system, also named $\mathrm{T}$, emerges. The system $\mathrm{T}$ is referentially decidable with respect to a frame which has an $\mathrm{R}$ with the following very simple characteristic: for all $\mathrm{w}$ in $\mathrm{W}$ : wRw. The condition is called reflexivity since what it amounts to is the condition that every world should have access to itself. One can prove that whereas reflexivity entails seriality, entailment does not work the other way around: seriality does not entail reflexivity. This means that the system $\mathrm{T}$ is contained in $\mathrm{D}$ and that the $\mathrm{R}$ 
in the frame for $\mathrm{T}$ in addition to being reflexive will also be serial. If you compare the definition of seriality with that of reflexivity, you will soon discover reflexivity to be but a special case of seriality: if every $w$ has access to itself, then every $\mathrm{w}$ also fulfils the condition that there is at least one $\mathrm{w}^{\prime}$ in $\mathrm{W}$ such that $w R w^{\prime}$, namely w itself (when $\left.w^{\prime}=w\right)$.

These few examples should suffice to give the reader a first impression of the flexibility of a Kripke frame. In my search for a decidable theory of meaning scepticism, I could not help becoming impressed by the richness of structures that a Kripke frame allows you to define simply in terms of one dyadic relation. It is clear that my problems as a sceptic would be solved the moment I managed to define a Kripke frame that could represent the four main characteristics of meaning listed in the previous section. For, if such a frame could be devised, there would also be a modal system that would be referentially decidable with respect to that frame. And since that modal system would be grammatically decidable as well, we would have a formal system in which to speak with absolute precision and decidability about a structure that - according to my stipulations - represented the main tenets of my theory of meaning. So, could a suitable Kripke frame be found, I would thereby have produced a very effective solution to the problem of expressing a theory of language scepticism without incurring the familiar paradoxes or inconsistencies.

As we know, a Kripke frame is just a pair $\langle\mathrm{W}, \mathrm{R}\rangle$, where $\mathrm{W}$ is a set of so-called worlds and $\mathrm{R}$ a dyadic relation on $\mathrm{W}$ (that is between the worlds of $\mathrm{W}$ ). We need not care that much about what worlds are. What we wish is to find a structure between these worlds that may represent the structure of meaning. However, for the record, let us think of a world as a collection of physical items. Each item may be thought of as representing some individual meaning. Since meanings, according to my theory, cannot be individually identified, we have to make sure that the structure we are looking for precludes any such identification. One of the first steps towards this goal is to ensure that no representative of meaning is contained in two or more worlds. So all worlds are different in the rather strong sense that each world contains a completely new stock of items. With these stipulations, we may think of $\mathrm{W}$ as representing some universe of meanings. 
We now need to structure this universe. We want the universe to be organised in a fashion that makes meanings come out as unrepeatable, finite, causal and temporal units, and we want to represent these four features by means of restrictions on $\mathrm{R}$. We start by letting $\mathrm{R}$ represent the relation that enables language users to process (have, produce, receive) meanings. We now ask which conditions must be placed on $\mathrm{R}$ for $\mathrm{R}$ to represent meaning processing as a unique, finite, causal and temporal affair.

Meaning processing must be represented as unique in the sense that it should not be possible to process the same meaning twice (or more). No world contains any item that is also contained in some other world; so if $\mathrm{R}$ represents meaning processing it should be clear that $\mathrm{R}$ will always take its user to ever new meanings. However, there is one leak. We know what reflexivity means: it amounts to the condition on $\mathrm{R}$ that worlds have access to themselves. Now, if $\mathrm{R}$ were reflexive, then $\mathrm{R}$ would represent a meaning-processing structure where users could have (could process) the same meaning twice (or more). So if $\mathrm{R}$ were reflexive, worlds would be accessible from themselves and it would be possible for $\mathrm{R}$ to represent a kind of processing of meaning where one and the same meaning could be processed more than once. In that case, meanings would fail to come out as unique and unrepeatable events. However, if we arrange for $\mathrm{R}$ to be irreflexive, we are safe. Irreflexivity, together with the absolute uniqueness of worlds, gives us what we want: a precise representation of the unrepeatability of meanings.

Now for the second feature: finiteness. $W$ is supposed to represent some semantic universe of real language users; thus, clearly, finiteness would be a natural restriction to impose on R. No language user has access to an infinite number of meanings. Since $\mathrm{W}$, according to my materialistic creed, is finite both with respect to the numbers of its worlds and to the numbers of items in each world, finiteness will, prior to any structuring of $\mathrm{R}$, be a feature of any Kripke frame I use. We might agree to settle for this kind of built-in finiteness; however, since I want to make $\mathrm{R}$ bear as much of the representative burden as possible, I shall look for a restriction on $\mathrm{R}$ that has the same effect.

There is a restriction known as converse well-foundedness ${ }^{27}$. It amounts to the condition that any subset of worlds from $\mathrm{W}$ must contain at least one last world, 
i.e., a world without successor in that subset. Since all of $\mathrm{W}$ is likewise a subset of $\mathrm{W}$, converse well-foundedness also requires that the whole of $\mathrm{W}$ contains some last world (or more, eventually). Above, when exemplifying the referential interrelationship between systems and frames, I mentioned the condition known as seriality. If $\mathrm{R}$ is serial, every world precisely has access to at least one more world. So, converse well-foundedness is in some sense the opposite of seriality ${ }^{28}$.

It should be evident that the structure of converse well-foundedness is incompatible with $\mathrm{R}$ having to structure an infinity of worlds. In such a situation (which I, for one, cannot imagine), there would be no dead ends, since infinity would demand ever new successors. Apart from being incompatible with infinity, converse well-foundedness has another agreeable incompatibility. It also excludes reflexivity. For if $\mathrm{R}$ were reflexive, then all w's would have access to themselves, and so there would be no worlds without successors. Under the condition of reflexivity, every world becomes its own successor. Under the condition of converse well-foundedness, some world has no successor.

According to my Spinozist interpretation, causality and temporality turn out to be structurally similar aspects of meaning ${ }^{29}$. In meaning processing, cause and effect are linked together in the same fashion as before and after. No cause of meaning is simultaneous with its effects, nor posterior to the same. Effects of meaning follow their causes just as one moment of meaning may be said to follow the next. So in claiming meanings to occur in a causal and temporal manner, I merely want to emphasise that meanings, albeit mental units, does not behave any differently from how we may believe their neuro-physiological counterparts to behave.

Logicians have been dealing with the combination of causality and temporality under the heading of determinism ${ }^{30}$. The issue is rather complicated and bristles with philosophical implications for which we have little use in this connection. What we are after is a condition on $\mathrm{R}$ that will impose a strict before-and-after (cause-and-effect) ordering on W. In the discipline known as temporal logic, the question of how to represent the flow of time has, naturally, come to the fore ${ }^{31}$. Especially two sets of conditions on $\mathrm{R}$ have attracted the interest of the craft: one known as weak linearity, the other sometimes referred to as discrete linearity. They 
both have the effects of stringing out all members of $\mathrm{W}$ on one single line (hence the name linearity). The difference is that while the weak version may allow for simultaneity (i.e., clusters of worlds all accessible from one single preceding world), its discrete analogue excludes any such clustering of worlds. Among logicians, the first kind of ordering is normally assumed to represent the flow of time as a continuous affair, the latter as a discrete one (hence my nomination). From our point of view, however, there is a serious problem with both. They both imply reflexivity, that is, the possibility that some world may be its own successor.

One may wonder why temporal logicians have come to believe that the flow of time includes the structure of reflexivity. The answer has to do with the way temporal logic emerged as a discipline ${ }^{32}$ and need not detain us here, especially since there exists a version of linearity, known as strict linearity, which is explicitly irreflexive. Like the two other forms of linearity, strict linearity organises its set of worlds along one line, but without clusters (simultaneity) and, as indicated, without reflexivity. More formally, by strict linearity one understands a dyadic relation $\mathrm{R}$ that in addition to being irreflexive is characterised by two conditions known as transitivity and weak connectedness.

Strict linearity comes in a finite as well as an infinite version. We, of course, choose the finite one. Irreflexivity is already part of our conditioning of R. Both finiteness and irreflexitivity are included in the condition of converse wellfoundedness. But what about transitivity and weak connectedness? How do they agree with converse well-foundedness?

Transitivity is the condition that if $\mathrm{wRw}^{\prime}$ and $\mathrm{w}^{\prime} \mathrm{Rw}^{\prime \prime}$, then we must also have ${ }^{w R w} w^{\prime \prime}$. If one world has access to another world with access to a third, then the first world must likewise have access to the third. Such are the workings of transitivity. I shall not go into the details of what transitivity does to the universe of meanings, the issue being thoroughly discussed in Nielsen (2003). Let me indicate instead how transitivity may be proved to be included in the condition of converse well-foundedness.

Remember that we work protected by the orderliness of grammatical and referential decidability. This means that the grammar of modal systems and 
conditions on $\mathrm{R}$ mirror each other in a completely uniform fashion (more specifically it means that for every condition on $\mathrm{R}$ there exists a modal system such that its theses are precisely those expressions that become valid on a frame where $\mathrm{R}$ has the condition in question). So when inquiring into whether two (or more) conditions on $\mathrm{R}$ may be compatible, one may seek an answer either by reasoning directly about $\mathrm{R}$, or by studying the modal systems that mirror these conditions.

Above, I showed reflexivity to be but a special case of seriality by directly reasoning about the features of R. I know of no similar arguing in the case before us, but fortunately a purely grammatical proof exists to the effect that converse well-foundedness implies transitivity. Since the proof is well suited for demonstrating how grammatical proofs work, I have included it in the appendix.

So, transitivity merely forms a natural part of what converse well-foundedness demands. That leaves us with weak connectedness. Will this also be compatible with converse well-foundedness? By connectedness is meant the condition on $\mathrm{R}$ that no two worlds accessible from a third may fail to be related by R. More formally: if $\mathrm{wRw}^{\prime}$ and $w \mathrm{Rw}^{\prime \prime}$, then either $\mathrm{w}^{\prime} \mathrm{Rw}^{\prime \prime}$ or $\mathrm{w}^{\prime \prime} \mathrm{Rw}^{\prime}$ (or both). The weakening of the condition consists in allowing $\mathrm{w}^{\prime}$ and $\mathrm{w}^{\prime \prime}$ to be one and the same world, in which case the condition merely states that no two worlds may fail to be directly related by $\mathrm{R}$. As with transitivity and converse wellfoundedness, I am not familiar with any semantic proof (proof by reasoning on R) to the effect that weak connectedness should be compatible with converse well-foundedness. But again, we have grammatical proofs to help us out. One may prove that a grammatically decidable modal system exists that is obtained by adding the characteristic axiom of weak connectedness to the modal system characterised by converse well-foundedness ${ }^{33}$. And since one may further show that the resulting system is characterised by a relation $\mathrm{R}$ combing converse wellfoundedness with weak connectedness, we may be certain that also weak connectedness complies with converse well-foundedness. In logic, one does not define impossible relations.

So, a finite strict linear ordering of worlds is a structure on $\mathrm{W}$ uniting the conditions of irreflexivity, finiteness, transitivity, converse well-foundedness and 


\section{K. HVIDTFELt NieLSEN}

weak connectedness. Let me summarise these five formal conditions in the following four traits:

1. No world has access to itself.

2. The number of worlds in $\mathrm{W}$ (under $\mathrm{R}$ ) is finite.

3. If any two worlds are successors of a third one, then one of these two worlds will be the successor of the other.

4. The ordering does not allow branching.

With this ordering, I take $\mathrm{R}$ to represent the structure of meaning as it is argued and explained in my book. The first trait tells us that meanings are unrepeatable; the second one assures us that meanings are finite in number. We may regard the third trait as an indication that cause and effect will prevail between any two instances of meaning; and by means of the last trait, the temporal structure of meanings should be rendered: time does not branch off in different directions, but remains an ordered flux.

Having found a formal representation of the way I believe (and have argued) meanings to be structured, I have finally come in full view of an effective solution to the problem driving my enterprise. With this paper (as with my book Nielsen 2003), I have wanted to show that the position of epistemic language scepticism need entail neither textual nor performative inconsistency. There is a way of speaking absolutely consistently of natural language scepticism without, at the same time, contradicting the position argued; it takes us beyond the limits of natural language, but that is as should be expected. What is more surprising is, perhaps, the fact that formal techniques may be adapted to suit the expressive needs of language theory. Having identified and defined a Kripke frame encapsulating those features of natural language meanings that, in my opinion, foster scepticism, we may now direct our attention towards the modal formal system characterised by that frame. If we could find a formal system decidably connected to the Kripke frame, we could use that system to speak with ultimate precision about the structure representing the general structure of meaning. As it turns out, my Kripke frame is in fact connected by referential decidability to a grammatically decidable modal system, namely, the system one obtains by adding the axioms known as $\mathrm{K}$ (for Kripke), $\mathrm{W}$ (for well-ordering) and D1 ${ }_{0}$ (D for 
Diodorus) to the non-modal propositional system (see the appendix and note 32).

This system, call it KWD1, has many interesting features of its own. To mention only one example, it contains, as a proper part, the system $\mathrm{KW}$ - also known as GL - which is known to be a modal version of the very system PA $^{34}$ that Gödel once proved to be grammatically undecidable ${ }^{35}$. In Nielsen (2003), I thoroughly discuss the special technique that Gödel devised for his proof, showing that his crucial results for PA do not carry over to its modal counterpart KW (and thus not to $\mathrm{KWD} 1_{0}$ ). Had KWD1 $1_{0}$ turned out to be grammatically undecidable, the whole idea of my enterprise would, of course, have been ruined. Personally, I find it thought provoking that the formal system in which to speak decidably of meaning turns out to contain, as a proper part, a modal version of the very system that Gödel proved to be undecidable. However, had my modal version of Gödel's system inherited the grammatical undecidability of the original, I would have met with quite a remarkable misfortune - or learned an even more extraordinary lesson.

Considering my scheme of using formal language in order to avoid the undecidabilities of natural language, it would have been disastrous to my whole project had the formal system I ended up with turned out to be provably undecidable. On the other hand, since mathematicians look at PA as the formal system encapsulating the essence of number theory, this misfortune might have induced me to speculate on a possible similarity between the undecidabilities of meanings and of numbers. I might have interpreted my failure as the proof that the common KW part of meaning theory and number theory would, in the grammars of both systems, engender the same kind of undecidability.

However, such are mere speculations. The system I identified as a possible theory of meaning is perfectly decidable, both grammatically and referentially. The system does have an interesting affinity to number theory. I do believe that the processing of meaning bears a structural relationship to the ordering of numbers. The correspondence may be banal or prove illuminating to linguists and language theorists. Only time can tell. But that is beyond the present point. My ambition has been to show that there exists a perfectly decidable way of 
expressing language scepticism. And, with all due modesty, I think I achieved my goal.

\section{LITERATURE}

Apel, Karl-Otto (1973), „Das Apriori der Kommunikationsgemeinschaft“, in: Apel, Karl-Otto (1973), Transformation der Philosophie, Bd. 2, Frankfurt am Main: Suhrkamp, pp. 358-435.

Boolos, George (1993), The Logic of Provability, Cambridge: Cambridge University Press.

Chellas, Brian F. (1980), Modal Logic. An Introduction, Cambridge: Cambridge University Press.

Church, Alonzo (1956), Introduction to Mathematical Logic, Princeton: Princeton University Press.

Frege, Gottlob (1962), Funktion, Begriff, Bedeutung, Göttingen: Vandenhoeck \& Ruprecht.

Frege, Gottlob (1966), Logische Untersuchungen, Göttingen: Vandenhoeck \& Ruprecht.

Gödel, Kurt (1931), „Über formal unentscheidbare Sätze der Principia mathematica und verwandter Systeme I", in: Gödel, Kurt (1986), Collected Works, vol. 1, New York: Oxford University Press, pp. 144-195.

Hasle, Per, Peter Øhrstrøm (1995), Temporal Logic. From Ancient Ideas to Artificial Intelligence, Dordrecht: Kluwer Academic Publishers.

Hooker, Michael (1980), „The Deductive Character of Spinoza's Metaphysics”, in: Kennington, Richard (ed.) (1980), The Philosophy of Baruch de Spinoza, Washington: Catholic University of America Press, pp. 17-34.

Hughes, G.E., M.J. Cresswell (1968), An Introduction to Modal Logic, London: Methuen.

Hughes, G.E., M.J. Cresswell (1984), A Companion to Modal Logic, London: Methuen.

Kripke, Saul A. (1963[1971]), „Semantic Considerations on Modal Logic“, in: Linsky, Leonard (ed.) (1971), Reference and Modality, Oxford: Oxford University Press, pp. 63-72.

Lemmon, E.J., Dana Scott (1977), An Introduction to Modal Logic, Oxford: Basil Blackwell.

Luhmann, Niklas (1980[1993]), Gesellschaftsstruktur und Semantik. Studien zur Wissenssoziologie der modernen Gesellschaft, Bd.1, Frankfurt am Main: Suhrkamp.

Luhmann, Niklas (1998), Die Gesellschaft der Gesellschaft, 2 Bde., Frankfurt am Main: Suhrkamp.

Montague, Richard (1974), Formal Philosophy, Yale: Yale University Press.

Nielsen, K. Hvidtfelt (2003), Interpreting Spinoza's Arguments. Toward a Formal Theory of Consistent Language Scepticism, Lewiston: Edwin Mellen Press. 
Nielsen, K. Hvidtfelt (2005), „Braucht eine Textlinguistik Kategorien des Sinns? Sinnkritische Bemerkungen zu Frege, Coseriu und Luhmann“, Tidsskrift for Sprogforskning, 3/1 (2005), pp. 47-83.

Prior, Arthur N. (1957), Time and Modality, Oxford: Clarendon.

Prior, Arthur N. (1967), Past, Present and Future, Oxford: Clarendon.

Spinoza, Baruch de (1677[1925]), Ethica. Ordine geometrico demonstrata, Heidelberg: Carl Winter.

Strauss, Leo (1948), „How To Study Spinoza's Theologico-Political Treatise”, in: Strauss, Leo (1952), Persecution and the Art of Writing, Chicago: Glencoe, pp. 142-201.

\section{NOTES}

1 The notion of performative (in)consistency was first discussed, if not actually named, in Apel (1973).

2 Nielsen (2003)

3 This assumption has been contested. In Strauss (1948), the philosopher Leo Strauss forcefully argues that Spinoza wrote his treatises with a view to more strategic intentions than those of pure cognition. A specimen of language scepticism, Strauss's unorthodox reading is discussed in Nielsen (2003).

4 See for instance Hooker (1980).

5 In Gödel (1931).

6 For a discussion of decidability in terms of referential concepts such as satisfiability and validity, see Church (1956).

7 In future references to formal systems and structures, the qualification physical will be dropped, but is, unless otherwise indicated, to be tacitly assumed.

8 By parsing the logician refers to more or less the same kind of operations as does the linguist by grammatical analysis. Under the condition of grammatical decidability, any expressions may be uniquely assigned to a grammatical category, also expressions that are not well-formed (grammatically impermissible combinations of words and operators) will be uniquely assigned to a category: the category of all malformed expressions.

9 That is of course without the restrictions of finiteness and physicality.

10 The difference between grammatical proofs and non-grammatical proofs is discussed in Nielsen (2003).

11 The story of decidability started with a non-grammatical proof of grammatical proofs: Gödel proved, in Gödel (1931), in a non-grammatical way that formal systems of a certain kind will contain sentences that are undecidable in the sense that neither the sentences themselves, nor their negations can be grammatically proved.

12 For a full and very readable (also for non-logicians) discussion of the notions of soundness and completeness, see Hughes and Cresswell (1984). In addition to the semantic kind of completeness, a grammatical version also exists. In this paper, only semantic completeness will be discussed.

13 The modern tradition of studying truth under the aspect of reference may be said to have originated with Frege (see Frege 1962 and 1966, papers originally published in the late $19^{\text {th }}$ century). In the following century, Church (1956) and Montague (1974) have 


\section{K. HVIDTFELT NieLSEN}

been among the foremost spokesmen of Frege's view. In Nielsen (2003), I give a detailed discussion of how to make formal truth a matter of denotation.

14 The word cenonomy is constructed, in analogy with synonymy, homonymy, etc., from the Greek kenos and onoma.

15 Synonymy is to be understood here in a referential sense. Since reference, in a logician's setting, is supposed to be a function, homonymy is excluded (remember that no function may take one and the same argument, now to one value, now to another, as homonymy would demand). A reference function without synonymy is a one-to-one function, with synonymy being a many-to-one function.

16 Instead of speaking of a frame, one may also speak of a class of models. See Hughes and Cresswell (1984) for an almost pedagogical introduction to the subject.

17 Reference is ultimately a question of tying formal systems and models together. So, in order to indicate that the link of models is missing, one often uses the locution of a reference on a frame. The 'on' is to be understood as implicating the need to establish reference by means of all those models that one may construct on (the ground of) some frame.

18 No observer can tell which meanings are occurring without 'having' the observed entities himself. So observers change by observing meanings. Another way of putting this odd predicament is to say that observation of meaning equals understanding (that is, as long as one foregoes a strictly behaviouristic approach to meaning). For a discussion of this intricate (and balefully philosophical) question, see Nielsen (2003) and Nielsen (2005). Incidentally (or perhaps not so incidentally), the very same question looms large in Luhmann's system theory (see Luhmann 1980[1993] and Luhmann 1998).

19 Another course open to students of cenonymy would be to keep silent. Mystics confronted with the impossibility of speaking about the ineffable have sometimes solved the problem by developing a highly eloquent kind of sigetics.

20 On the interdependency of grammatical and referential decidability, see note 24 .

21 In logic, one makes the distinction between a model for - and a model of something. The first locution is used when speaking about the relationship between a formal system and a formal model. A model is a model for some formal system when soundness between model (the frame on which the model may be constructed) and system prevails, i.e., when all theses of the system have valid reference in the model and on the frame. The second locution refers to the normal idea of a model as some kind of representation of a segment of reality.

22 One of the reasons why modal quantificational systems are so hard to interpret stems from the need to deal with two kinds of quantification: one over worlds and another over items in the respective worlds.

23 Apart from the situations where two (or more) modal axioms can be shown to be equivalent. See Chellas (1980) for a synoptic view of such situations.

24 All systems to be discussed in this section are grammatically decidable. Notice that grammatical decidability is a necessary prerequisite for referential decidability: one could not identify theses with valid expressions without first identifying the theses uniquely.

25 When naming formulae and systems, I usually follow Hughes and Cresswell (1984); only occasionally, I resort to the naming practice of Lemmon and Scott (1977).

26 According to Hughes and Cresswell (1968: 31), the system T was so baptised in 1953 by the logician Sobicinski.

27 The relation converse well-foundedness as well as the reasons for choosing this name are thoroughly discussed in Boolos (1993). 
28 In spite of converse well-foundedness being opposed to seriality, we do not get seriality just by turning converse well-foundedness around. The converse of converse wellfoundedness, that is well-foundedness, is a condition on $\mathrm{R}$ that makes any subset of worlds from $\mathrm{W}$, ordered by $\mathrm{R}$, contain some first world, that is, a world from which no other worlds (in that subset) are accessible. So whereas converse well-foundedness excludes seriality, well-foundedness is compatible with seriality, yet without implying it.

29 In Ethica, Spinoza distinguished between three orders of cognition: the order of affects (merely a pre-form of cognition), the order of rational cognition and the order of eternal cognition. Naturally, causal and temporal structures cease to conflate when cognition operates under the third kind of orders. In Nielsen (2003), I identify Spinoza's second order with the kind of cognition that we expect from science today. So, under the order of science, causality and temporality should run parallel.

30 Spinoza believed the whole universe, including the thoughts and behaviour of man, to obey strict deterministic laws.

31 For a recent survey of temporal logic, see Hasle and Øhrstrøm (1995).

32 Prior, the founding father of temporal logic, took as his starting point an interpretation of Aristotle made by the Greek philosopher Diodorus (the third century B.C.). Diodorus had suggested that Aristotle's modal concepts necessity and possibility be translated into true now and ever after and true now or sometimes later. Through this mixture of epistemic (truth-related) and purely temporal conditions, Diodorus, and with him Prior - together with most of contemporary temporal logic, made reflexivity part of temporal concerns. Suppose we took the operators $\square$ and $\diamond$ to express Diodorus's idea of time. And suppose we were to evaluate an expression like $\square \alpha$ in the Kripke way by demanding $\square \alpha$ to be true with respect to some world w only if $\alpha$ were true with respect to all worlds $\mathrm{w}^{\prime}$ accessible from w. Which worlds would they be? Since $\square$ is to mirror the idea of a proposition being true now and evermore, then clearly $\alpha$ would also have to be true with respect to $\mathrm{w}$ itself (the 'now' world, as one might say), and so $\mathrm{R}$ would have to be reflexive. By the same reasoning one realises that also $\diamond$ must be interpreted by a reflexive $\mathrm{R}$.

33 An informal sketch of this proof is found in Hughes and Cresswell (1984: 104f.).

34 PA stands for Peano Arithmetic; actually Gödel used a modified version of Russell's logical system from Principia mathematica; see Gödel (1931).

35 Boolos (1993) gives a full (if condensed) presentation of how to transform Gödel's reasoning and logic into modal terms and symbolism.

\section{APPENDIX}

The characteristic axiom of normal modal systems:

$\mathrm{K}: \square(\mathrm{A} \rightarrow \mathrm{B}) \rightarrow(\square \mathrm{A} \rightarrow \square \mathrm{B})$

The characteristic rule of normal modal systems:

$\mathrm{N}$ : if $\alpha$ is a thesis, so is $\square \alpha$

The characteristic axiom of seriality:

$\mathrm{D}: \square \mathrm{A} \rightarrow \diamond \mathrm{A}$ 


\section{K. HVIDTFELT NieLSEN}

The characteristic axiom of reflexivity:

$\mathrm{T}: \square \mathrm{A} \rightarrow \mathrm{A}$

(There are no characteristic axioms of non-seriality og irreflexivity, so these features can only be indirectly denoted)

The characteristic axiom of transitivity:

4: $\square \mathrm{A} \rightarrow \square \square \mathrm{A}$

The characteristic axiom of converse well-foundedness:

$\mathrm{W}: \square(\square \mathrm{A} \rightarrow \mathrm{A}) \rightarrow \square \mathrm{A}$

The characteristic axiom of strict linearity:

$\mathrm{D} 1_{0}: \square((\square \mathrm{A} \wedge \mathrm{A}) \rightarrow \mathrm{B}) \vee \square((\square \mathrm{B} \vee \mathrm{B}) \rightarrow \mathrm{A})$

The modal system KWD1 $1_{0}$ is obtained by adding to the non-modal propositional system (=PS) the three axioms $\mathrm{K}, \mathrm{W}$ and $\mathrm{D} 1_{0}$ and the rule $\mathrm{N}$ of all normal modal systems. From PS, N, K and W, axiom 4 follows.

The grammatical proof that PS, N, K and W imply 4:

(1) $\quad \mathrm{A} \rightarrow((\square \square \mathrm{A} \wedge \square \mathrm{A}) \rightarrow(\square \mathrm{A} \wedge \mathrm{A}))-$ a substitution instance of the PS thesis $\mathrm{p}$ $\rightarrow((\mathrm{r} \wedge \mathrm{q}) \rightarrow(\mathrm{q} \wedge \mathrm{p}))$; substitute A for $\mathrm{p}, \square \mathrm{A}$ for $\mathrm{q}$ and $\square \square \mathrm{A}$ for $\mathrm{r}$.

(2) $\quad \mathrm{A} \rightarrow(\square(\square \mathrm{A} \wedge \mathrm{A}) \rightarrow(\square \mathrm{A} \wedge \mathrm{A}))$ - obtained from (1) by applying the modal equivalence ( $\square \square \mathrm{A} \wedge \square \mathrm{A}) \leftrightarrow \square(\square \mathrm{A} \wedge \mathrm{A}$ ) (the modal equivalence follows from PS, $\mathrm{N}$ and $\mathrm{K})$.

(3) $\square(\mathrm{A} \rightarrow(\square(\square \mathrm{A} \wedge \mathrm{A}) \rightarrow(\square \mathrm{A} \wedge \mathrm{A})))$ - obtained from (2) by applying $\mathrm{N}$.

(4) $\quad \square \mathrm{A} \rightarrow(\square(\square(\square \mathrm{A} \wedge \mathrm{A}) \rightarrow(\square \mathrm{A} \wedge \mathrm{A}))$ - obtained from (3) by first substituting in $\mathrm{K}$ the formula $(\square(\square \mathrm{A} \wedge \mathrm{A}) \rightarrow(\square \mathrm{A} \wedge \mathrm{A}))$ for $\mathrm{B}$ and then applying modus ponens (if $\alpha$ and $\alpha \rightarrow \beta$ are theses, then $\beta$ is likewise one (modus ponens: a rule of PS)); the substitution makes $\mathrm{K}$ have the form $(3) \rightarrow(4)$, and since $\mathrm{K}$ and (3) are theses, (4) is likewise one by modus ponens.

(5) $\quad \square(\square(\square \mathrm{A} \wedge \mathrm{A}) \rightarrow(\square \mathrm{A} \wedge \mathrm{A})) \rightarrow \square(\square \mathrm{A} \wedge \mathrm{A})-$ a substitution instance of W; substitute $(\square \mathrm{A} \wedge \mathrm{A})$ for $\mathrm{A}$.

(6) $\quad(\square \mathrm{A} \rightarrow \square(\square(\square \mathrm{A} \wedge \mathrm{A}) \rightarrow(\square \mathrm{A} \wedge \mathrm{A}))) \rightarrow(\square(\square(\square \mathrm{A} \wedge \mathrm{A}) \rightarrow(\square \mathrm{A} \wedge \mathrm{A})) \rightarrow$ $\square(\square \mathrm{A} \wedge \mathrm{A})) \rightarrow(\square \mathrm{A} \rightarrow \square(\square \mathrm{A} \wedge \mathrm{A}))-$ a substitution instance of the PS thesis $(\mathrm{p} \rightarrow \mathrm{q}) \rightarrow((\mathrm{q} \rightarrow \mathrm{r}) \rightarrow(\mathrm{p} \rightarrow \mathrm{r}))$; substitute $\square$ A for $\mathrm{p}, \square(\square(\square \mathrm{A} \wedge \mathrm{A}) \rightarrow$ $(\square \mathrm{A} \wedge \mathrm{A}))$ for $\mathrm{q}$, and $\square(\square \mathrm{A} \wedge \mathrm{A})$ for $\mathrm{r}$.

(7) $\quad \square \mathrm{A} \rightarrow \square(\square \mathrm{A} \wedge \mathrm{A})$ - obtained from (4), (5) and (6) by first realising that (6) has the form $(4) \rightarrow((5) \rightarrow(7))$ and then applying modus ponens twice, first detaching (4) and then detaching (5). 
(8) $\square \mathrm{A} \rightarrow(\square \square \mathrm{A} \wedge \square \mathrm{A})$ - obtained from (7) by applying the modal equivalence $(\square \square \mathrm{A} \wedge \square \mathrm{A}) \leftrightarrow \square(\square \mathrm{A} \wedge \mathrm{A}$ ) (see under (2)).

(9) $(\square \square \mathrm{A} \wedge \square \mathrm{A}) \rightarrow \square \square \mathrm{A}-$ a substitution instance of the PS thesis $(\mathrm{p} \wedge \mathrm{q}) \rightarrow$ p; substitute $\square \square$ A for $\mathrm{p}$ and $\square$ A for $\mathrm{q}$.

$(10) \quad(\square \mathrm{A} \rightarrow(\square \square \mathrm{A} \wedge \square \mathrm{A})) \rightarrow((\square \square \mathrm{A} \wedge \square \mathrm{A}) \rightarrow \square \square \mathrm{A}) \rightarrow(\square \mathrm{A} \rightarrow \square \square \mathrm{A}))-\mathrm{a}$ substitution instance of the PS thesis $(p \rightarrow q) \rightarrow((q \rightarrow r) \rightarrow(p \rightarrow r))$; substitute $\square$ A for $\mathrm{p}, \square \square \mathrm{A} \wedge \square$ A for $\mathrm{q}$ and $\square \square \mathrm{A}$ for $\mathrm{r}$.

(11) $\square \mathrm{A} \rightarrow \square \square \mathrm{A}$ - obtained from (8), (9) and (10) by first realising that (10) has the form $(8) \rightarrow((9) \rightarrow(11))$ and then applying modus ponens twice, first detaching (8) and then detaching (9). - QED. 